\title{
The SARS-CoV-2 variant, Omicron, shows rapid replication in human primary nasal epithelial cultures and efficiently uses the endosomal route of entry.
}

Thomas P. Peacock ${ }^{1 \#}$, Jonathan C. Brown ${ }^{1 \#}$, Jie Zhou ${ }^{1 \#}$, Nazia Thakur², Joseph Newman², Ruthiran Kugathasan ${ }^{1}$, Ksenia Sukhova ${ }^{1}$, Myrsini Kaforou ${ }^{1}$, Dalan Bailey ${ }^{2}$, and Wendy S. Barclay ${ }^{1 *}$

"These authors contributed equally to this work

${ }^{1}$ Department of Infectious Disease, Imperial College London, UK, W2 1PG

${ }^{2}$ The Pirbright Institute, Woking, Surrey, UK, GU24 ONF

*Corresponding author: w.barclay@imperial.ac.uk

\section{Abstract}

At the end of 2021 a new SARS-CoV-2 variant, Omicron, emerged and quickly spread across the world. It has been demonstrated that Omicron's high number of Spike mutations lead to partial immune evasion from even polyclonal antibody responses, allowing frequent re-infection and vaccine breakthroughs. However, it seems unlikely these antigenic differences alone explain its rapid growth; here we show Omicron replicates rapidly in human primary airway cultures, more so even than the previously dominant variant of concern, Delta. Omicron Spike continues to use human ACE2 as its primary receptor, to which it binds more strongly than other variants. Omicron Spike mediates enhanced entry into cells expressing several different animal ACE2s, including various domestic avian species, horseshoe bats and mice suggesting it has an increased propensity for reverse zoonosis and is more likely than previous variants to establish an animal reservoir of SARS-CoV-2. Unlike other SARSCoV-2 variants, however, Omicron Spike has a diminished ability to induce syncytia formation. Furthermore, Omicron is capable of efficiently entering cells in a TMPRSS2-independent manner, via the endosomal route. We posit this enables Omicron to infect a greater number of cells in the respiratory epithelium, allowing it to be more infectious at lower exposure doses, and resulting in enhanced intrinsic transmissibility. 


\section{Introduction}

The second year of the SARS-CoV-2 pandemic was marked by the repeated emergence of SARS-CoV-2 'variants' with altered antigenicity, and/or enhanced transmissibility. The fifth 'variant of concern', Omicron (Pango lineage BA.1), was first detected in Southern Africa ${ }^{1}$ and, within a just a month of its initial detection, has rapidly spread across the world comprising the majority of SARS-CoV-2 cases in many countries including the UK ${ }^{2}$. Omicron is marked by a total of 51 mutations across the entire genome including an unprecedented 33 mutations in its Spike glycoprotein gene $\mathrm{e}^{1,3}$. The resulting large antigenic distance between Omicron and the Wuhan-like Spike protein used in current vaccines ${ }^{3-9}$, correlates with a lower vaccine effectiveness against Omicron and a higher frequency of reinfections ${ }^{2,10,11}$. However, it is unclear if vaccine breakthrough and reinfections alone fully account for Omicron's rapid growth and displacement of the Delta variant, or whether an inherent increase in ability to infect the human airway may also contribute to the rising case numbers.

\section{Results}

Omicron replicates more rapidly in primary cultures of human nasal epithelial cells but is attenuated for entry in many cell lines

In Vero E6 cells overexpressing human ACE2 and TMPRSS2 protease (Vero-AT cells) ${ }^{12}$, both Delta and Omicron variants formed plaques, but the plaques for Omicron were much smaller and less distinct (Figure 1A).

The virus stocks of each variant contained similar viral RNA-to-infectivity ratios as measured by E gene viral load and plaque titres. Equal titres of each variant were used to inoculate trans-wells of primary cultures of human nasal epithelial cells (hNECs), or infect Vero E6-ACE2/TMPRSS2 (Vero-AT) cells, or Calu-3 cells, an immortalised human lung cell that expresses abundant ACE2 and TMPRSS2 ${ }^{13}$. Virus released at the apical surface of the airway cultures, or into the supernatants overlaying the monocultures was collected daily for 3 days post-inoculation and viral loads determined by RT-qPCR for E gene and by plaque assay for infectious virus on Vero-AT cells (Figure 1B). In hNECs Omicron showed a large early replication advantage, yielding viral titres $\sim 100$-fold higher than Delta by 24 hours postinfection. At 48 and 72 hours post-infection, viral titres were lower compared to Delta and at 72 hours the RNA collected from Omicron infected wells contained fewer E gene copies. This 
coincided with observations by light microscopy at 72 hours that Omicron-infected wells displayed complete ciliostasis, suggesting cell death, whereas beating cilia were still observed in the Delta-infected wells. In Vero-AT cells, replication of the two variants was equal despite the observation that Omicron formed smaller plaques in these cells (Figure 1A). In Calu-3 cells the viral yields of Omicron were lower than for Delta across all time points.

A
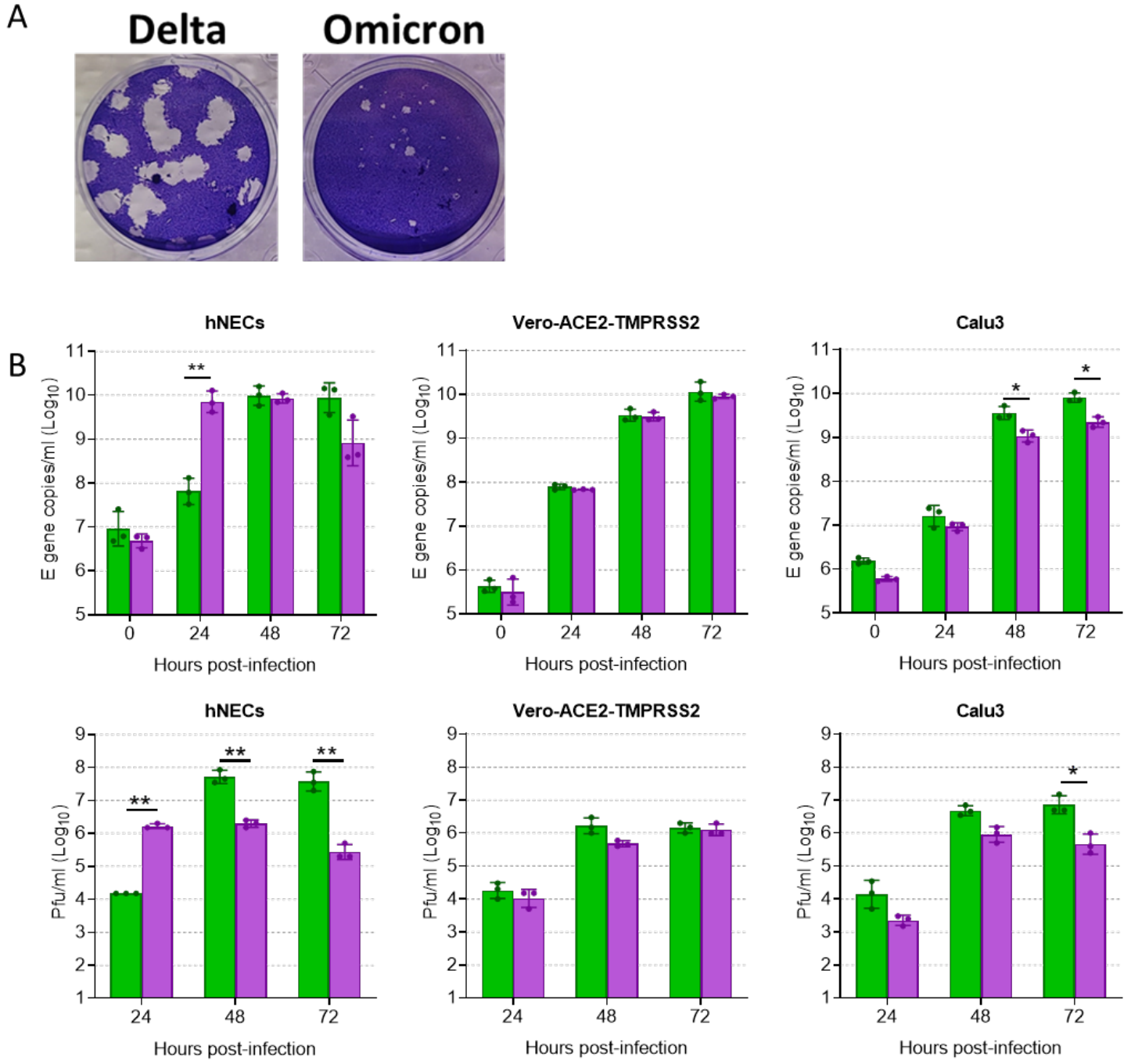

- Delta 1

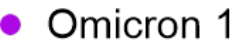

Figure 1 - Comparative replication kinetics of Omicron and Delta. (A) Virus isolates were propagated on Vero-ACE2-TMPRSS2 (VAT) cells and plaqued on VAT cells for 3 days before visualising by crystal violet staining. (B) Comparative replication kinetics of SARS-CoV-2 Omicron and Delta variants in vitro and ex vivo. Primary human nasal epithelial cultures (hNECS) were inoculated with Delta or Omicron variants at $0.1 \mathrm{pfu} / \mathrm{cell}$, and Vero-ACE2-TMPRSS2 (Vero-AT) and Calu-3 cells were inoculated at 0.001 $\mathrm{pfu} / \mathrm{cell}$. Infections were performed in triplicate wells and back titration confirmed equal inputs of the variants by E gene copies measured by RT-qPCR and infectious units measured by plaque assay. Bars show individual replicate titres with the standard deviation. Statistical differences measured by ANOVA on log transformed data. *, $\mathrm{P}<0.05 ;{ }^{* *}, \mathrm{P}<0.01 ;{ }^{* * *}, \mathrm{P}<0.001 ; * * * *, \mathrm{P}<0.0001$ 


\section{Omicron outcompetes Delta in primary cultures of human nasal airway epithelial cells}

We next performed a mixed competition assay inoculating an equal amount of each variant into the same wells of hNECs, Vero-ATs, or Calu-3 cells. Once again virus released from the apical surface of the primary cultures, or into the supernatant overlaying the immortalised cells, was collected daily and assessed for total virus replication by E gene RT-qPCR (dotted black lines in Figure 2A) and also for the RNA yield for each variant using a novel variantspecific PCR protocol taking advantage of a unique insertion/deletion pair present or absent in the aa210-215 region of Omicron or Delta Spike (See Supplementary Figure S1A). We first validated the specific RT-qPCR by creating a series of artificial mixes of RNA from each variant (Supplementary Figure S1B). During mixed infection of primary hNECs, the Omicron isolate rapidly outcompeted Delta so that it was the only virus yielding detectable RNA products from apical washes from 24 hours post-infection. In contrast, both viruses in mixed infections remained in similar quantities in the Vero-AT cells, and in Calu-3 cells Delta outcompeted Omicron and dominated by 72 hours post-infection (Figure 1A). Importantly, we repeated the competition experiment using a completely different pair of Delta vs Omicron isolates (Figure 1B). The second Delta isolate was of the sublineage AY.4.2, which was emerging and outcompeting other Delta sublineages in the UK in Autumn $2021^{14}$. As before, AY.4.2 was rapidly outcompeted by the second isolate of Omicron in the primary hNECs, but not in VeroAT. In Calu-3, AY.4.2 rapidly outcompeted the Omicron isolate, again suggesting a severe attenuation of Omicron in these cells. 
A

hNECs

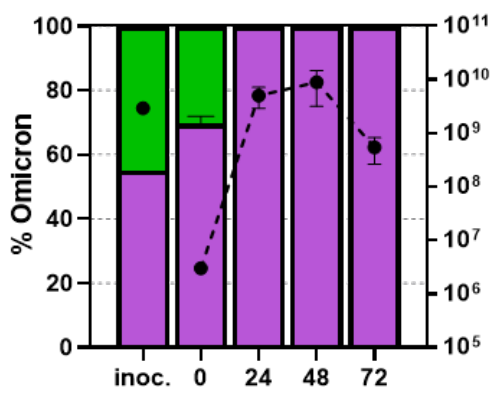

Hours post-infection

B

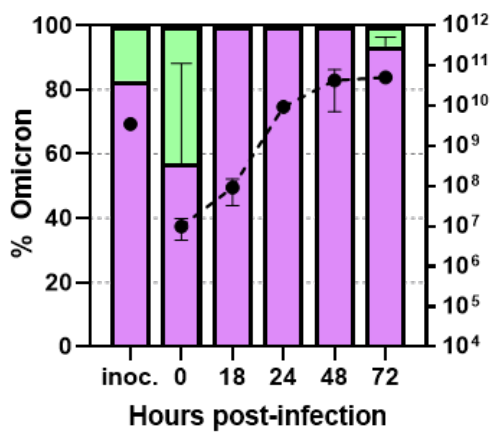

Vero-ACE2-TMPRSS2

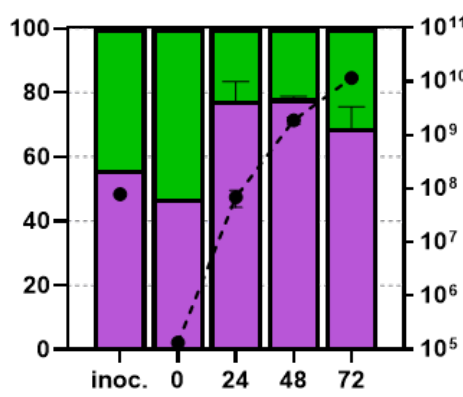

Hours post-infection
Calu-3

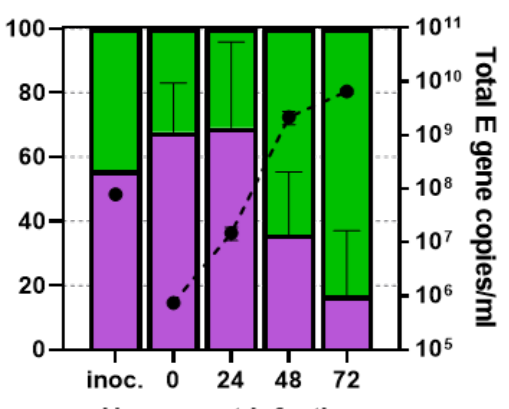

Hours post-infection
Vero-ACE2-TMPRSS2

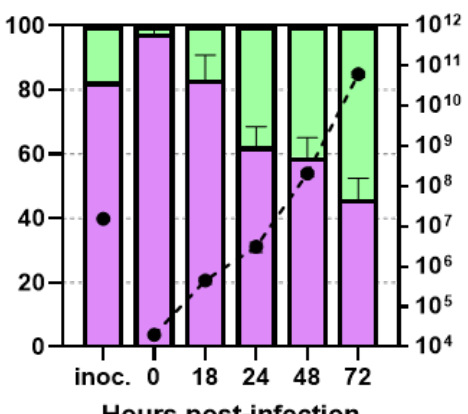

Calu-3

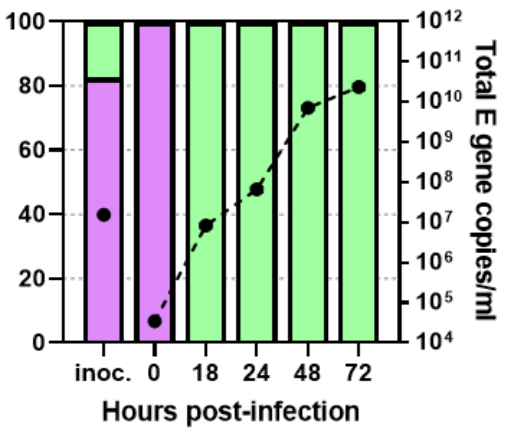

Figure 2-Competition assays between Delta and Omicron isolates. $(A, B)$ Competition assay between SARS-CoV-2 Omicron (Purple) and Delta (Green) isolates in vitro and ex vivo. A probe-based RT-qPCR assay was developed to measure the proportion of Omicron and Delta present in mixed samples (see Supplementary Figure S1). The proportion of variant-specific signal is shown with the SD of 3 replicates. (A) Equal mixtures or (B) an 8:2 ratio of Omicron and Delta isolates were inoculated onto hNECs at a final multiplicity of $0.1 \mathrm{pfu} / \mathrm{cell}$ and onto Vero-AT and Calu-3 cells at $0.001 \mathrm{pfu} / \mathrm{cell}$. Daily harvests were measured for total viral load by E gene qRT-PCR (black dots) and by the variant specific RT-qPCR to give variant proportions. Mean + s.d. of 3 replicates shown. (A) Shows competition assay specifically between BA.1 (Spike A701V) and a B.1.617.2 isolate while (B) shows competition between a different BA.1 isolate (lacking A701V) an AY.4.2 isolate.

\section{Omicron continues to use ACE2 for cell entry rather than other protein receptors}

One hypothesis to explain why Omicron shows such rapid replication in nasal cells is that, due to its large number of receptor binding domain (RBD) mutations, Omicron might use a different, or a wider range of protein receptors such as those used by other seasonal or epidemic coronaviruses. To test this, we investigated entry of Omicron Spike-containing lentiviral pseudotypes into 293T cells expressing different canonical human coronavirus receptors including angiotensin-converting Enzyme 2 (ACE2), aminopeptidase N (APN), or dipeptidyl peptidase-4 (DPP4) (Figure 3A). While pseudoviruses expressing the Spike proteins 
of 229E and MERS entered more efficiently when their canonical receptors APN or DPP4, respectively, were expressed, NL63 and all SARS-CoV-2 variants, including Omicron, only entered efficiently when ACE2 was overexpressed, implying this remains the canonical protein receptor for Omicron, as has been confirmed by others ${ }^{3}$.

We further confirmed the interaction between Omicron Spike protein and human ACE2 using a flow cytometry-based method to measure the interaction between soluble human ACE2 and different membrane bound Spike proteins (Figure 3B). As this assay is based on expressing whole Spike on the surface of HEK 293Ts and measuring binding to soluble, fluorescent ACE2 it has the advantage of being able to incorporate the impact of dynamic conformational changes in Spike, unlike RBD-only biophysical methods. Whole Omicron Spike was found to bind to human ACE2 to a greater extent than the Spike proteins of either Alpha or Delta variants. An Alpha Spike engineered to contain the additional substitution Q498R, however, displayed similar binding to Omicron Spike suggesting the combination of N501Y and Q498R in Omicron Spike might be responsible for its enhanced binding to human ACE2.

\section{Omicron shows an extended ACE2 host range above other variants}

Substitutions within the Spike RBD have been shown to alter the host-tropism of SARSCoV-2, leading to enhanced replication in mice, ferrets, or mink ${ }^{15-17}$. Separately, recent studies from the US have established that white-tailed deer can act as a reservoir for the virus ${ }^{18}$, highlighting a potential future issue for the pandemic. The risk of reverse zoonosis combined with the ongoing high case numbers of this variant led us to investigate whether the hostrange of Omicron is significantly different. Pseudovirus bearing Omicron Spike was markedly more able to enter cells using the ACE2 proteins from several species not used efficiently by other SARS-CoV-2 variants, notably domestic poultry, horseshoe bats, and mice (Figure 3C, Supplementary Figure S2). Indeed using the flow cytometry-based binding assay described above we found the full length Omicron Spike protein bound murine ACE2 much more efficiently than even Alpha Spike (Figure 5D), which also contains known mouse adaptation N501 $\mathrm{Y}^{19}$. 
A

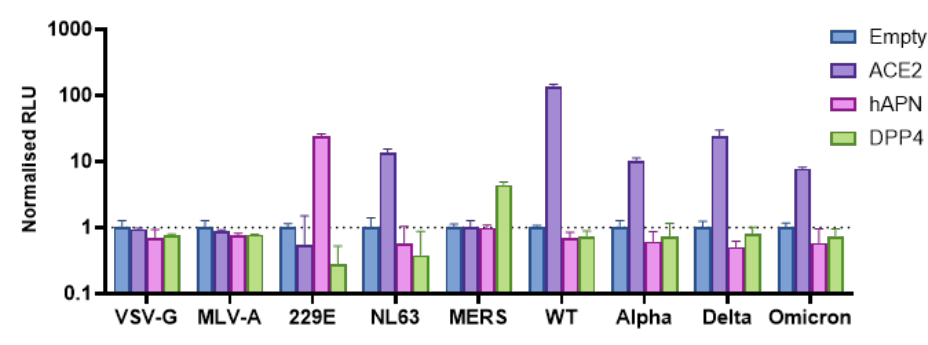

C

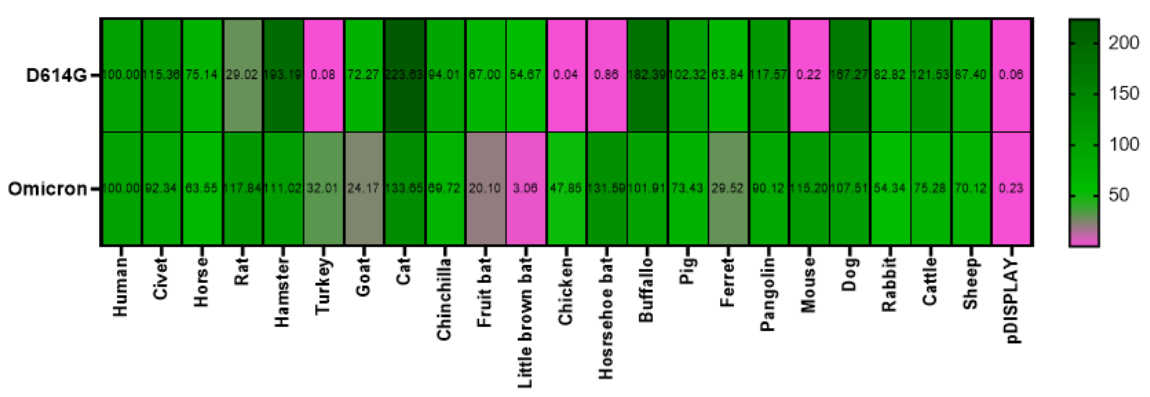

B

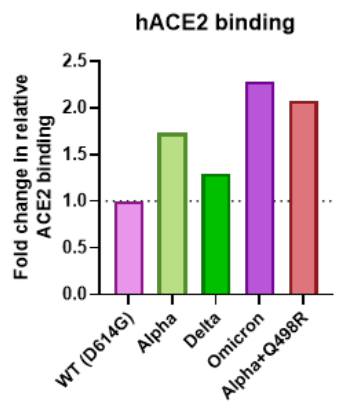

D

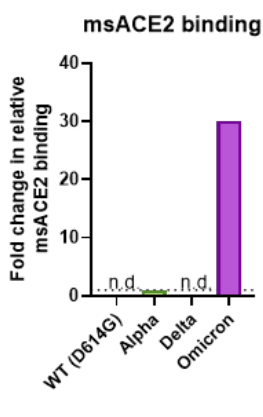

Figure 3 - Use of common coronavirus protein receptors and receptor species specificity for SARSCoV-2 variant entry. (A) Entry of pseudotyped lentiviruses expressing different viral glycoproteins was assessed into 293Ts transfected with different coronavirus protein receptors. 229E and MERS Spike protein pseudotypes were used as controls for hAPN and DPP4 mediated entry. All assays were performed in triplicate and are plotted as mean + s.d. (B, D) HEK 293T cells expressing whole SARSCoV-2 Spike were incubated with recombinant (B) hACE2-Fc(IgG) for 30 minutes or (D) mouse ACE2$\mathrm{Fc}(\operatorname{lgG})$ overnight, washed and incubated with Goat Anti-Human IgG Fc (DyLight ${ }^{\circledR} 650$ ) secondary for 30 minutes, before washing and analysis by flow cytometry. Relative differences in median fluorescence intensity are shown. 'n.d.' indicates not detected. (C) Receptor usage was screened using pseudoviruses expressing the indicated Spike proteins into BHK-21 cells expressing the indicated ACE2 protein. Viral entry was measured by assaying luciferase activity (RLU) using the BrightGlo reagent (Promega).

\section{Despite containing mutations that enhance Spike cleavage, Omicron causes reduced syncytia formation compared to previous SARS-CoV-2 variants}

SARS-CoV-2 Spike proteins from first wave isolates and all subsequent VOCs induces syncytia formation when expressed on the surface of cells and mixed with target cells expressing ACE2. Efficient syncytia formation, as well as cell surface entry of SARS-CoV-2, requires Spike to have been pre-cleaved in producer cells at a multibasic furin cleavage site at the S1/S2 junction. The Delta variant Spike mutation, P681R, is known to both enhances cleavage and also results in increased syncytia formation ${ }^{20-22}$. Omicron contains two 
mutations adjacent to the furin cleavage site, $\mathrm{N} 679 \mathrm{~K}$ and $\mathrm{P} 681 \mathrm{H}$, that have the potential to increase furin cleavage above that seen for the wild type Spike (Figure 4A). Introduced individually into a plasmid expressing the wild type Spike (D614G), each of these mutations did indeed result in increased S1/S2 cleavage (Figure 4B,C). Puzzlingly, whole Omicron Spike did not mediate efficient cell fusion with 293T or BHK target cells compared to either D614G or Delta Spike which both gave highly efficient syncytia formation (Figure 4D, E). This suggests the cell surface fusion of Omicron was attenuated compared to previous strains, despite carrying mutations that should enhance cleavage. 
A

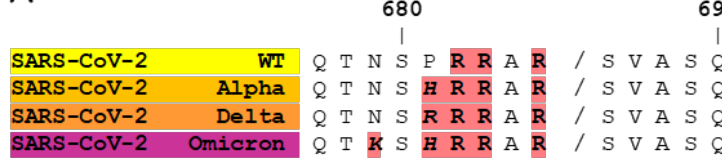

B

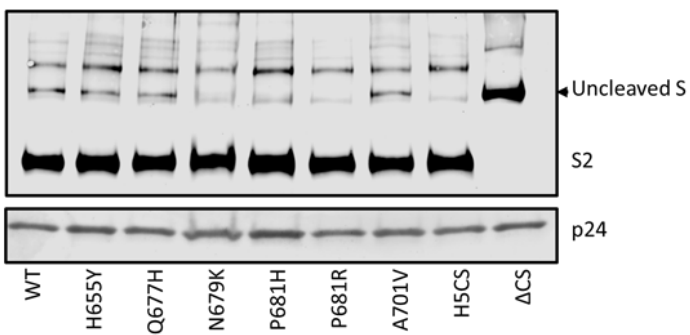

SARS-CoV-2 Spike D614G

D

$293 T$

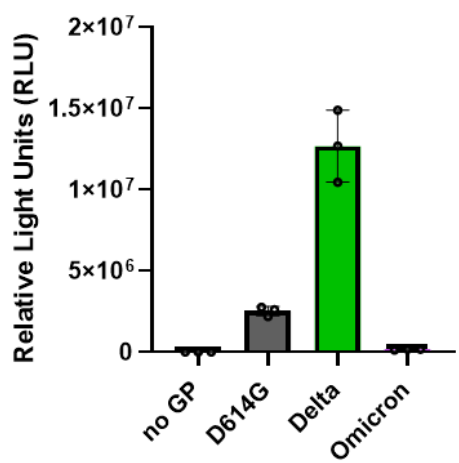

$\mathrm{E}$

Empty vector
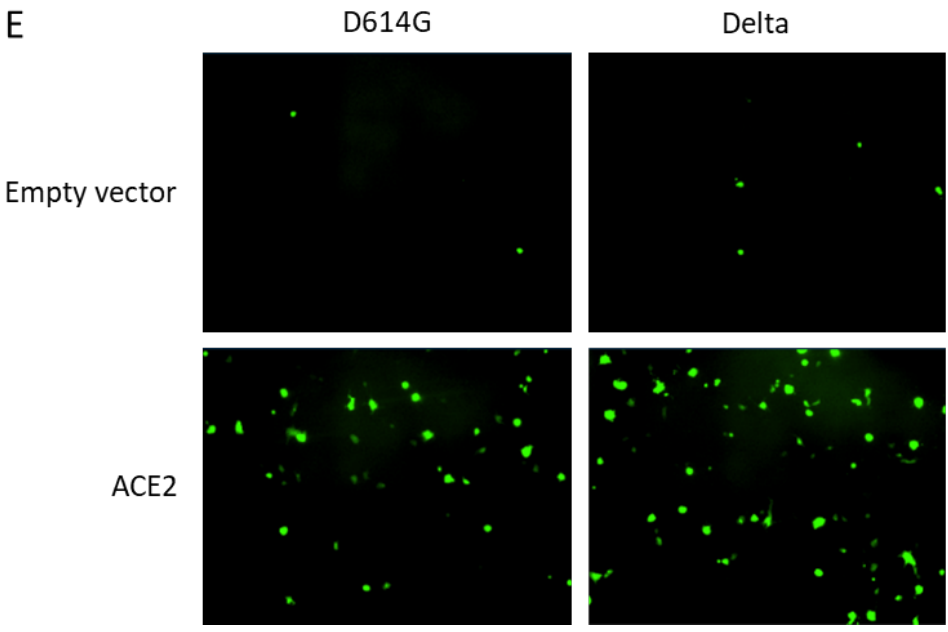

C

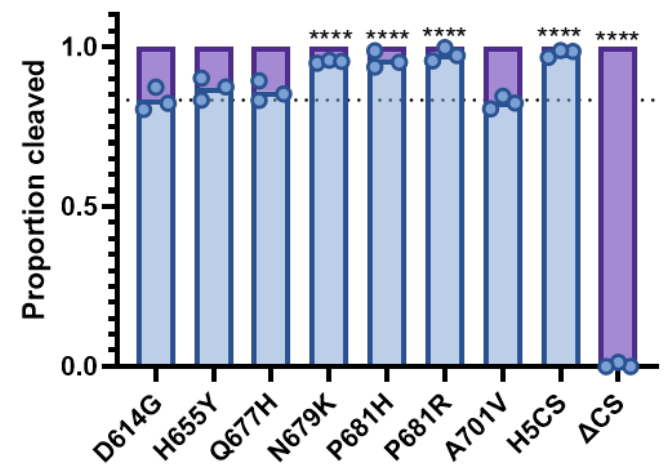

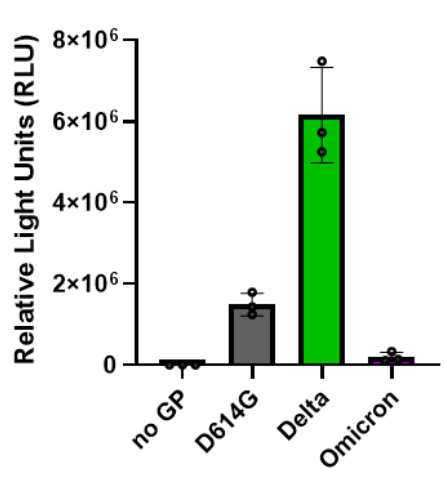

Delta
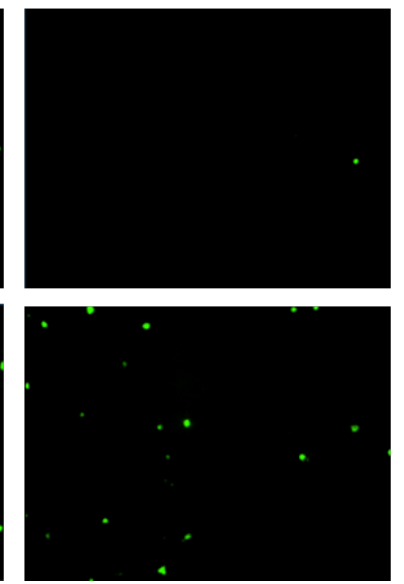

Figure 4-Cleavage and syncytia formation assays of Omicron Spike. (A) Schematic of S1/S2 cleavage site of Spike protein from different variants. Basic residues highlighted in red. (B) Western blot analysis of concentrated pseudovirus expressing different SARS-CoV-2 Spike mutants. Levels of lentiviral p24 shown as loading control. Representative blot of $\mathrm{N}=3$ repeats. (C) Western blot densitometry of cleavage of mutants from part (B), N=3, data plotted as mean + s.d. Statistical differences measured by one-way ANOVA with multiple comparisons to WT. *, P<0.05; **, $\mathrm{P}<0.01 ; * * *, \mathrm{P}<0.001 ; * * * *$, $P<0.0001$ (D) Cell-cell fusion assays of HEK 293T or BHK21 cells with rLUC-GFP1-7 expressing the stated Spike protein and BHK-21 cells expressing human ACE2 and rLUC-GFP 8-11. All assays were performed in triplicate and are plotted as mean + s.d. (E) representative repeat of fusion assay from part (D) on 293T. 


\section{Omicron can efficiently enter cells by the endosomal entry pathway}

To determine if Omicron was using a different, or additional cell entry pathways we measured pseudovirus entry into several different cell lines - 293T-ACE2 cells, H1299s (nonsmall cell lung carcinoma cells), Caco-2 (immortalised human intestinal cells that express abundant ACE2 and TMPRSS2 ${ }^{13}$ ), and Calu-3 cells. In 293T-ACE2 and H1299 cells, pseudovirus bearing Omicron Spike mediated efficient entry, greater than that of Alpha or Delta Spike, however in Caco-2 and Calu-3 Omicron pseudovirus entered more poorly than all other variants (Figure $5 \mathrm{~A})$.

We and others have previously shown that, due to the presence of a furin cleavage site at the S1/S2 junction, early isolates of SARS-CoV-2 preferentially enter cells in a TMPRSS2dependent manner at the cell surface to avoid endosomal entry where they would be heavily restricted by innate factors such as IFITM proteins ${ }^{13,23,24}$. Omicron pseudovirus entered cells most efficiently that lack TMPRSS2 expression (293T-ACE2 and H1299) ${ }^{13,25}$, but was more attenuated for entry compared to other VOCs, in cells that express abundant TMPRSS2 (Calu3 and (Caco-2). To investigate whether Omicron was more efficiently entering cells via the endosomal route, we tested the relative sensitivity of pseudovirus bearing Omicron Spike to inhibitors of cell-surface entry (Camostat, a broad inhibitor of serine proteases such as TMPRSS2) or endosomal entry (E64d, an endolysosomal cathepsin inhibitor). We included a control pseudovirus with wild type Spike but lacking the S1/S2 furin cleavage site ( $\triangle C S$ ) which we previously showed preferentially enters cells via the endosome. In Caco-2 cells, which allow entry of SARS-CoV-2 in both a TMPRSS2-dependent or -independent manner ${ }^{13}$, Omicron $^{2}$ pseudovirus entry was more sensitive to E64d and less sensitive to Camostat than other variants, behaving in a manner more similar to $\Delta$ CS.

We further assessed any role for TMPRSS2 in mediating Omicron pseudovirus entry by introducing TMPRSS2 to 293Ts that otherwise lack endogenous expression ${ }^{13}$ (Figure 5C). Whereas TMPRSS2 expression extensively enhanced entry of pseudovirus bearing D614G, Alpha and Delta Spikes by 25-150 fold, Omicron pseudovirus entry was only modestly improved, increasing to a similar degree as $\Delta \mathrm{CS}$ ( $\sim 10$-fold). This suggests Omicron Spike is far 
less reliant on TMPRSS2 for cell entry and can enter some cell types more efficiently using the endosomal route.

We and others previously showed SARS-CoV-2 viruses that enter via the endosome are more sensitive to inhibition of entry endosomal IFITM restriction factors, such as IFITM2 $/ 3^{13,23}$. However, this restriction can be lifted by the addition of Amphotericin $B$ (AmphoB). In both Calu-3 and Caco-2 cells, entry of Omicron pseudovirus, as seen for pseudovirus with the $\triangle C S$ Spike, was significantly enhanced by the addition of AmphoB, whereas other variants and WT Spike were not (Figure 5D).

A

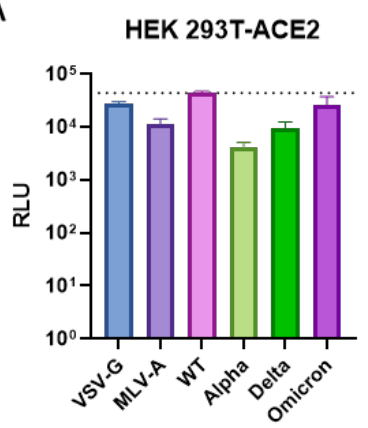

B

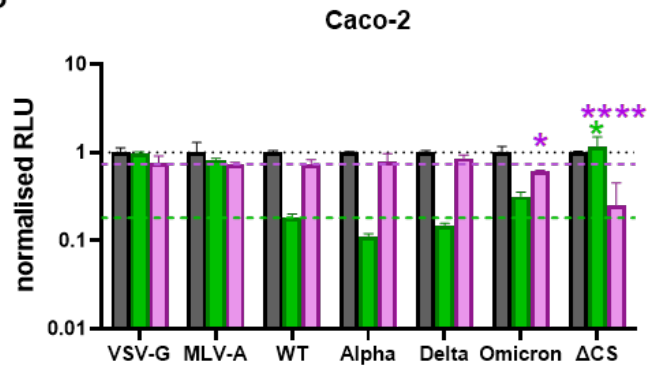

Caco-2

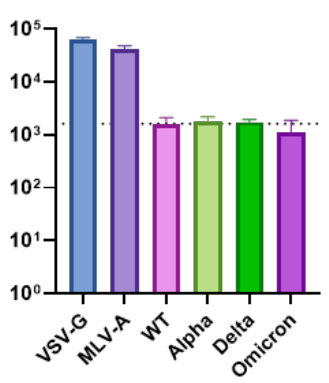

C

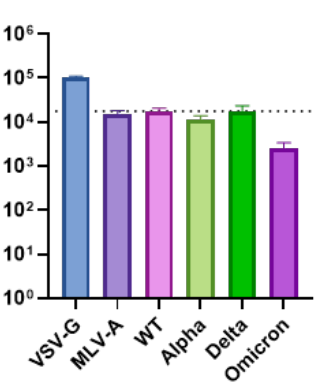

Calu-3

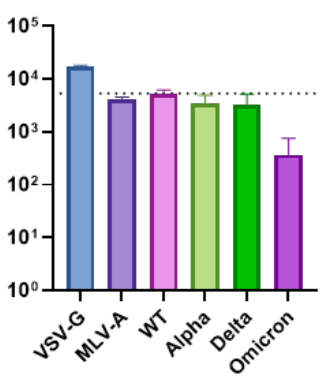

$293 T+A C E 2 \pm$ TMPRSS2

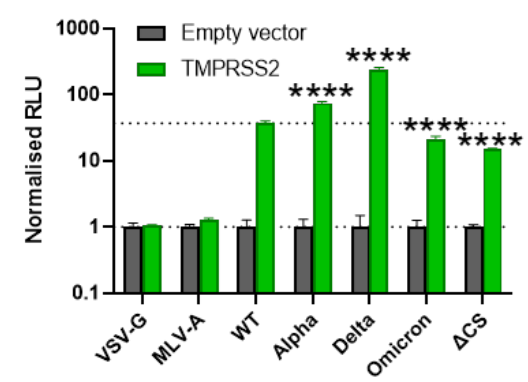

Calu-3
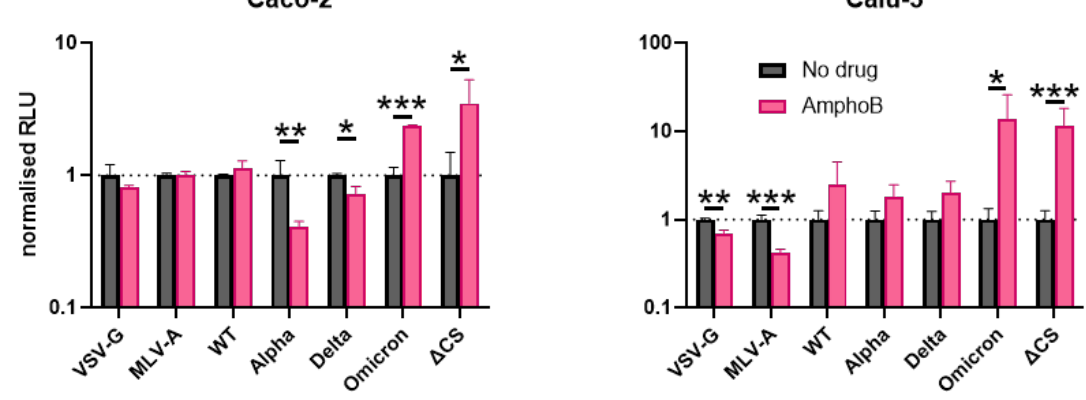

Figure 5 - Cell entry mechanism of Omicron. (A) Cell entry of pseudoviruses containing different viral glycoproteins into 293T-ACE2, H1299, Caco-2 or and Calu-3 cells. Cells were transduced with different pseudoviruses and lysed $48 \mathrm{~h}$ later, and analysed for firefly luciferase luminescence. Data are shown as raw luminescence units (RLU). All assays were performed in triplicate and are plotted as mean + s.d. $(B, D)$ Inhibition/enhancement of entry of pseudoviruses expressing different viral glycoproteins into 
Caco-2 or Calu3 pretreated with (B) the serine protease inhibitor, camostat (green bars), or the cathepsin inhibitor, E64-d (pink bars), or (D) the endosomal IFITM restriction factor inhibitor Amphotericin B (bright pink bars). Assays were performed in triplicate and are plotted as mean + s.d. Data shown are a representative replicate $(n=3)$. All data were normalized to no drug control (grey bars). (C) Relative lentiviral pseudovirus entry into 293T cells expressing non-cleavable ACE2-C4$\mathrm{FLAG}^{26}$ with or without co-expression of TMPRSS2. Entry into cells transfected with empty vector instead of TMPRSS2 was normalized to 1 . Assays were performed in triplicate and are plotted as mean + s.d.

Finally, we investigated the entry route of live Omicron virus in primary human airway cells. hNECs were pretreated with Camostat or AmphoB and multicycle replication kinetics of Delta or Omicron isolates were compared. As before, in untreated hNECs Omicron virus replicated much more rapidly than Delta virus (Figure 6, Figure 1B). In the presence of Camostat, Delta virus replication was totally inhibited, with no detectable increase in titres during whole experiment, again confirming the complete dependence of this variant on serine proteases, such as TMPRSS2, for entry. In contrast, the Omicron isolate replicated in the presence of Camostat, eventually reaching equivalent titres to the control without the drug (Figure 6). The delay in replication in presence of Camostat confirms that although Omicron replication is enhanced by TMPRSS2, this pathway is not essential. Interestingly, the addition of AmphoB had little effect on the replication of either variant. This suggests that in the context of live virus infection in primary airway cells, neither Delta nor Omicron are substantially inhibited by the IFITM proteins. This is of particular interest as we previously showed considerable enhancement of virus replication following AmphoB treatment of airway cultures of an early Vero cell-adapted SARS-CoV-2 mutant lacking the furin cleavage site that entered cells exclusively via the endosome. It would appear that Omicron virus can now productively utilize the endosomal pathway whilst avoiding endosomal IFITM restriction. 

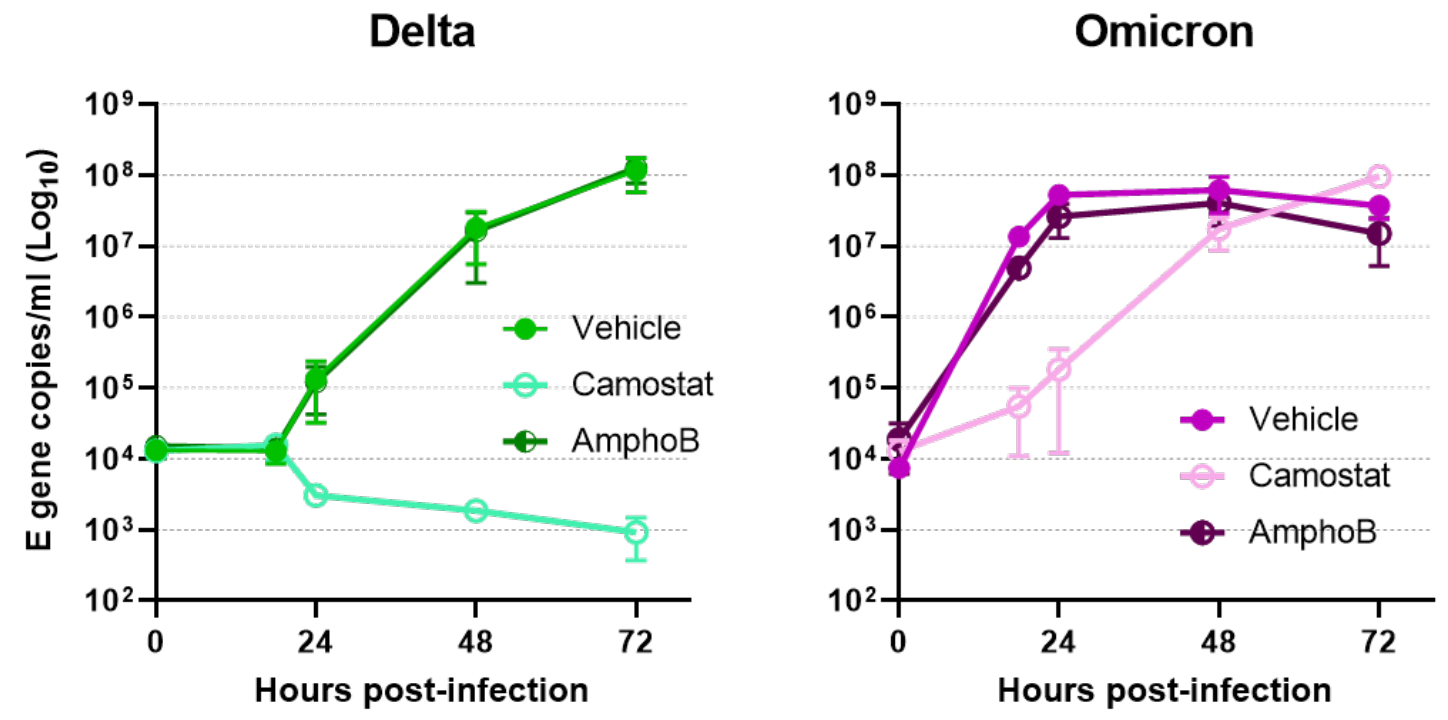

Figure 6 - Cell entry mechanism of live Omicron virus in primary cells. Comparative replication kinetics of SARS-CoV-2 Omicron and Delta variants in primary human nasal epithelial cultures (hNECs) inoculated with Delta or Omicron variants at $0.1 \mathrm{pfu} / \mathrm{cell}$. Cells were pretreated both basolaterally and apically with either with no drug, or with $50 \mu \mathrm{M}$ of Camostat or Amphotericin B for 2 hours prior to infection. 50 $\mathrm{MM}$ of Camostat or Amphotericin B remained in the basolateral media for the course of the experiment. Infections were performed in triplicate wells and back titration confirmed equal inputs of the variants by E gene copies measured by RT-qPCR and infectious units measured by plaque assay. Plotted as mean \pm s.d.

\section{Discussion}

Data on Omicron's global prevalence indicates this variant is one of the most rapidly spreading SARS-CoV-2 variants observed to date. Increased reinfection rates and vaccine breakthroughs undoubtably contribute to this rapid growth, but other factors such as an inherent increase in transmissibility could also be playing a part. Here we show that Omicron virus replicates extremely rapidly within primary cultures of nasal epithelial cells, even more so than Delta, which itself replicated faster than any previously characterised variants ${ }^{20}$. We propose that Omicron achieved this rapid replication rate by becoming less specialised in terms of its cellular tropism. Earlier SARS-CoV-2 isolates were limited in their tropism by their requirement for cell surface entry using the TMPRSS2 protease ${ }^{13,24}$, and it appears subsequent variants, such as Alpha and Delta, became even more heavily reliant. However only a low proportion of cells in the upper respiratory tract express both ACE2 and TMPRSS2 (Table 1$)^{27}$. Here we show that Omicron has adapted to additionally use the ubiquitous 
endosomal pathway for entry, thus expanding the number of cell types it can infect, as single cell RNA seq data indicates that seven times more cells in the nose express both ACE2 and the ubiquitous endosomal cathepsin proteases than co-express ACE2 and TMPRSS2 (Figure 7) ${ }^{27}$. Unlike previous SARS-CoV-2 mutants lacking the furin cleavage site, which used the endosomal pathway but were heavily restricted by endosomal restriction factors, such as IFITM proteins, Omicron appears refractory to this inhibition.

\begin{tabular}{|l|l|l|l|l|}
\hline Row Labels & ACE2+TMPRSS2- ACE2-TMPRSS2 & ACE2+TMPRSS2+ ACE2-TMPRSS2- \\
\hline Epithelial (Basal) & 25 & 286 & 4 & 4998 \\
\hline Epithelial (Ciliated) & 9 & 68 & 0 & 395 \\
\hline Epithelial (lonocyte) & 0 & 8 & 0 & 86 \\
\hline Epithelial (Secretory) & 20 & 226 & 4 & 1478 \\
\hline Epithelial (Serous) & 3 & 205 & 2 & 1737 \\
\hline Immune (Lymphocyte) & 1 & 23 & 0 & 3165 \\
\hline Immune (Myeloid) & 0 & 8 & 0 & 989 \\
\hline Stromal (Endothelial) & 1 & 14 & 0 & 1049 \\
\hline Stromal (Fibroblast) & 2 & 6 & 0 & 1416 \\
\hline Stromal (SMC) & 0 & 2 & 0 & 153 \\
\hline Total & $\mathbf{6 1}$ & $\mathbf{8 4 6}$ & $\mathbf{1 0}$ & $\mathbf{1 5 4 6 6}$ \\
\hline
\end{tabular}

Table 1. Single cell transcriptomic analysis of nasal epithelial cells from Muus et al ${ }^{27}$. 


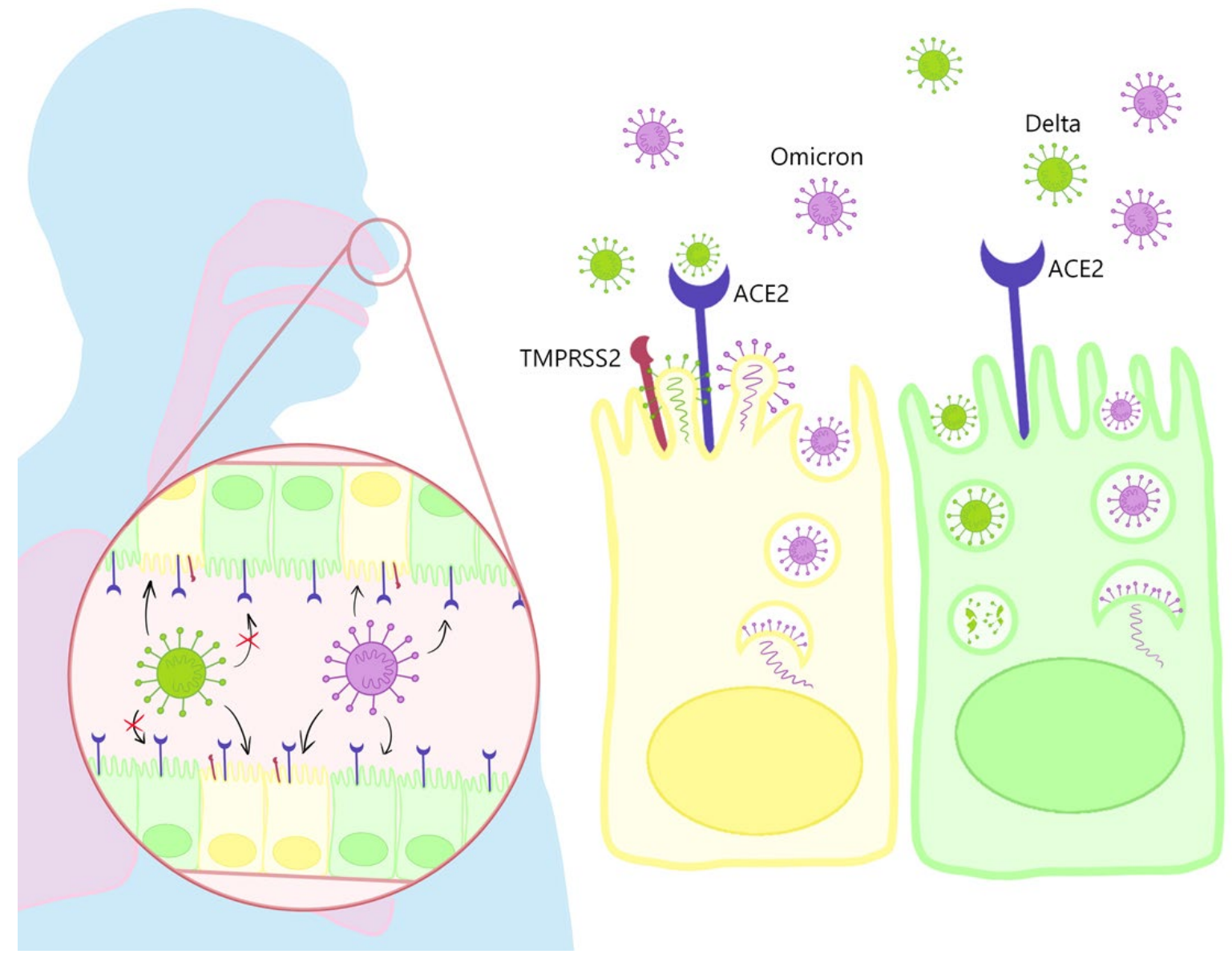

Figure 7 - Proposed model for Omicron's altered entry pathway leading to more rapid replication in the nasal epithelium. Previous SARS-CoV-2 isolates and variants, such as Delta, only enter cells efficiently by binding ACE2 and activating fusion via cell-surface protease TMPRSS2. This allows them to enter at the cell surface and bypass the endosome, where potent restriction factors, such as IFITM proteins, are enriched. Omicron, conversely, is able to enter cells in both a TMPRSS2-dependent and -independent manner, having evolved the ability to avoid endosomal restriction. This allows Omicron to infect any ACE2-expressing cell in the airway instead of relying solely on double $\mathrm{ACE}^{+} \mathrm{TMPRSS2}^{+}$ cells.

Whilst the switch in entry pathway has increased its inherent transmissibility, the less efficient use of cell surface TMPRSS2 by Omicron Spike protein has also resulted in a decrease in the propensity for syncytia formation, as we and others have now shown 28,29 . This in turn may decrease disease severity since syncytia have been reported in autopsy of COVID cases and the efficient cleavage at the furin site that underlies syncytia formation has been associated with enhanced disease severity in animal models ${ }^{21,30,31}$. Moreover, the contrast between the dominance of Delta virus in Calu-3 cells derived from a bronchial adenocarcinoma, vs Omicron virus in primary nasal airway cells suggests the Omicron variant 
may be more likely to replicate at the site of transmission and less so in the deeper lung, thus driving the virus towards efficient transmission and away from severe disease, as is now being seen in some animal models $29,32,33$.

In this study we found that the Omicron Spike protein resulted in a high ACE2 binding in the context of whole Spike protein, consistent with several other studies ${ }^{5,34,35}$, that may also contribute to increased transmission. In addition, we found Omicron Spike bound better to mouse ACE2 than any previous variant, as several others have reported ${ }^{5,35}$, as well as to the ACE2 proteins of some domestic avian species, and certain bat species. This leads to the possibility that the host range of Omicron may be widened even further giving it the potential for reverse zoonosis and establishment of novel wild or domestic animal reservoirs from which future SARS-CoV-2 variants or recombinants might emerge. However, whether ACE2 is the only species-specific factor that currently limits host range potential is not clear, for example we were previously unable to infect avian cells, even when transfected to express human $A C E 2^{36}$. This data may also give some tentative clues about any potential animal origins of Omicron which remains a popular hypothesis.

In summary the Omicron Variant of Concern demonstrates important biological changes compared to previous SARS-CoV-2 variants that may be leading to a more transmissible, but less inherently severe virus. Any decrease in inherent severity should be considered in the context of the extremely high cases numbers resulting from widespread transmission, which will lead inevitably to high health care burden as vulnerable individuals succumb. The emergence of Omicron variant underscores the continued need for vaccination strategies to keep pace with the virus evolution to limit spread and disease severity. Moreover, Omicron demonstrates high potential to establish a reservoir in other animals, and this makes it even less likely that SARS-CoV-2 will ever be eradicated.

\section{Materials and methods}

\section{Biosafety and ethics statement}

All laboratory work was approved by the local genetic manipulation safety committee of Imperial College London, St. Mary's Campus (centre number GM77), and the Health and Safety Executive of the United Kingdom, under reference CBA1.77.20.1.

\section{Cells}


Human embryonic kidney cells (293T; ATCC CRL-11268) were maintained in complete media (DMEM, 10\% FCS, 1\% non-essential amino acids (NEAA) and 1\% penicillin-streptomycin (P/S)). African green monkey kidney cells overexpressing human ACE2 and TMPRSS2 (Vero E6-ACE2-TMPRSS2; Glasgow University $)^{12}$, were maintained in complete media supplemented with $200 \mu \mathrm{g} / \mathrm{ml}$ hygromycin $B$ (Gibco) and 2mg/ml G418 (Gibco) to maintain human ACE2 and TMPRSS2 expression. Human lung cancer cells (Calu-3; ATCC HTB-55) and Human epithelial colorectal adenocarcinoma cells (Caco-2; ATCC HTB-37) were maintained in DMEM, 20\% FCS, 1\% NEAA and 1\% P/S. Human non-small cell lung Carinoma cells (NCI-H1299; ATCC CRL-5803) were maintained in RPMI-1640 medium, 10\% FCS, $1 \% \mathrm{NEAA}$, and $1 \% \mathrm{P} / \mathrm{S}$. Baby hamster kidney cells (BHK-21; ATCC CCL-10) were maintained in DMEM supplemented with 10\% FCS, 1 mM sodium pyruvate solution (Sigma-Aldrich, Germany), and 1× P/S. Air-liquid interface human nasal epithelial cells (hNECs) were purchased from Epithelix and maintained in Mucilair cell culture medium (Epithelix). All Cells were kept at $5 \% \mathrm{CO}_{2}, 37^{\circ} \mathrm{C}$.

\section{SARS-CoV-2 virus}

An Omicron isolate was kindly provided by Gavin Screaton Oxford University and propagated on Vero E6-ACE2-TMPRSS2 cells, in serum-free DMEM supplemented with $0.3 \%$ bovine serum albumin (BSA). Cells were incubated for 3 days until cytopathic effect was observed.

Upper respiratory tract swabs used to isolate viruses were collected for routine clinical diagnostic use and sequenced using the ARTIC network protocol (https://artic.network/ncov-2019) to confirm the presence of BA.1 lineage virus, under approval by the Public Health England Research Ethics and Governance Group for the COVID-19 Genomics UK consortium (R\&D NR0195). Delta and Omicron virus were isolated by inoculating $100 \mu \mathrm{L}$ of neat swab material onto Vero E6-ACE2-TMPRSS2 cells, incubating at $37^{\circ} \mathrm{C}$ for $1 \mathrm{~h}$ before replacing with growth media supplemented with $1 \mathrm{x}$ penicillin/streptomycin and $1 \mathrm{x}$ amphotericin B. Cells were incubated for 5-7 days until cytopathic effect was observed. Isolates were passaged a further two times in Vero E6-ACE2-TMPRSS2 cells ${ }^{12}$.

\begin{tabular}{|l|l|l|l|}
\hline Name in text & Virus name & PANGO lineage & GISAID/COG Accession \\
\hline Delta 1 & $\begin{array}{l}\text { hCoV-19/England/SHEF- } \\
10 E 8 F 3 B / 2021\end{array}$ & B.1.617.2 & EPI_ISL_1731019 \\
\hline Delta 2 & ISST987 & AY.4.2 & N/A \\
\hline Omicron 1 & M21021166 & B.1.1.529/BA.1 & N/A \\
\hline Omicron 2 & NWLP04 & B.1.1.529/BA.1 & PHEP-YYNTNOA \\
\hline
\end{tabular}

Table 1. Virus isolates used in this study. 
Virus growth kinetics and competition assays were performed as described previously ${ }^{13}$. Briefly, in air-liquid interface hNECs, before infection cells were washed with serum-free media to remove mucus and debris. Cells were infected with $200 \mu \mathrm{L}$ of virus-containing serum-free DMEM and incubated at $37^{\circ} \mathrm{C}$ for $1 \mathrm{~h}$. Inoculum was then removed, and cells were washed twice. Time points were taken by adding $200 \mu \mathrm{L}$ of serum-free DMEM and incubating for 10 mins and $37^{\circ} \mathrm{C}$ before removal and titration.

For SARS-CoV-2 plaque assays, serial dilutions of virus supernatant in serum-free DMEM, $1 \%$ NEAA and $1 \% \mathrm{P} / \mathrm{S}$ were performed and inoculated onto VAT cells for $1 \mathrm{~h}$ at $37^{\circ} \mathrm{C}$. Inoculum was then removed and replaced with SARS-CoV-2 overlay medium ( $1 \times \mathrm{MEM}, 0.2 \% \mathrm{w} / \mathrm{v} \mathrm{BSA}, 0.16 \% \mathrm{w} / \mathrm{v} \mathrm{NaHCO}$, $10 \mathrm{mM}$ HEPES, $2 \mathrm{mM}$ L-Glutamine, $1 \times \mathrm{P} / \mathrm{S}, 1 \% \mathrm{w} / \mathrm{v}$ Avicel). Plates were incubated for $3 \mathrm{~d}$ at $37^{\circ} \mathrm{C}$ before overlay was removed and cells were stained for $1 \mathrm{~h}$ at room temperature in crystal violet solution.

\section{Plasmids, pseudovirus and pseudovirus entry assays.}

Spike-encoding pcDNA3.1 plasmids were generated by mutagenesis or by gene synthesis as described elsewhere $\mathrm{e}^{4,37}$. Pseudovirus was generated and concentrated as previously described ${ }^{13}$. All spike expression plasmids used in this study contain D614G and K1255*STOP (that results in deletion of the C-terminal cytoplasmic tail of spike containing the endoplasmic retention signal, aka the $\Delta 19$ spike truncation). pCAGGs-ACE2-FLAG and non-cleavable pCAGGs-ACE2C4-FLAG were used a previously described ${ }^{13,38}$. TMPRSS2 expression plasmid was a gift from R. Reeves (Addgene plasmid no. 53887; http://n2t.net/addgene:53887; RRID: Addgene_53887 ${ }^{39}$.

Overexpression experiments in 293T cells were performed by co-transfecting either pCAGGSACE2-FLAG or non-cleavable pCAGGs-ACE2-C4-FLAG, with or without TMPRSS2 into $100 \mathrm{~mm}$ dishes of 293T cells. Controls were transfected with equal amounts of empty vector instead of the named plasmid. After $24 \mathrm{~h}$, cells were washed, resuspended into fresh media and added to PV, or spun down for analysis by western blot.

Species ACE2 entry assays were performed as previously described ${ }^{36}$. Briefly, BHK cells were transfected with $500 \mathrm{ng}$ of ACE2 or empty vector (pDISPLAY) using TransIT-X2 (Mirus Bio) according to the manufacturer's recommendation. $24 \mathrm{~h}$ later, media was removed, and cells were harvested following the addition of $2 \mathrm{mM}$ EDTA in PBS, resuspended in DMEM and plated into white-bottomed 96 wells plates (Corning). Cell were overlayed with pseudovirus and incubated for $48 \mathrm{~h}$. Firefly luciferase was quantified whereby media was replaced with $50 \mu \mathrm{L}$ Bright-Glo substrate (Promega) diluted 1:2 with PBS and read on a GloMax Multi+ Detection System (Promega).

\section{Cell-cell fusion assays}


Cell-cell fusion assays were performed as described elsewhere ${ }^{36,40}$. Briefly, 293Ts stably expressing rLuc-GFP 1-7 effector cells ${ }^{41}$ were transfected with empty vector, WT or mutant SARS-CoV2 spike proteins. 293T, BHK-21, or Calu-3 target cells stably expressing rLuC-GFP-8-11 (target cells) were co-transfected with ACE2 expression constructs. Target cells were co-cultured with effector cells $24 \mathrm{~h}$ post-transfection and quantification of cell-cell fusion was performed $24 \mathrm{~h}$ later with the Renilla luciferase substrate, Coelenterazine-H (Promega). Luminescence was read on a Glomax Multi+ Detection System (Promega).

\section{Flow cytometry-based Spike-ACE2 affinity assay}

HEK 293 T cells were transfected with mammalian expression plasmids encoding the relevant SARS-CoV2 spike protein. 24 hours after transfection, cells were dissociated and incubated with recombinant human ACE2-Fc(IgG) (1mg/ml) (Abcam ab273687) at a dilution of 1:32000 for 30 minutes or recombinant mouse ACE2 Protein, (His \& hFc Tag) (sinobiological 50249-M03H) at a dilution of 1:250 overnight. Following the incubation, cells were incubated with Goat Anti-Human IgG Fc (DyLight ${ }^{\circledR} 650$ ) preadsorbed (ab98622) for 30 minutes before determination of median fluorescence on a BD LSR Fortessa.

\section{Western Blotting}

Virus or pseudovirus concentrates were lysed in $4 x$ Laemmli buffer (Bio-rad) with $10 \% \beta$ mercaptoethanol and run on SDS-PAGE gels. After semi-dry transfer onto nitrocellulose membrane, samples were probed with mouse anti-p24 (abcam; ab9071) and rabbit anti-SARS spike protein (NOVUS; NB100-56578). Near infra-red (NIR) secondary antibodies, IRDye ${ }^{\circledR}$ 680RD Goat anti-mouse (abcam; ab216776) and IRDye ${ }^{\circledR}$ 800CW Goat anti-rabbit (abcam; ab216773) were subsequently used to probe membranes. Western blots were visualised using an Odyssey Imaging System (LI-COR Biosciences).

\section{Viral RNA extraction and E gene qPCR}

Virus genomes were quantified by E gene RT-qPCR as previously described ${ }^{42}$. Viral RNA was extracted from cell culture supernatants or hamster nasal washes using the QIAsymphony DSP Virus/Pathogen Mini Kit on the QIAsymphony instrument (Qiagen). RT-qPCR was then performed using the AgPath RT-PCR (Life Technologies) kit on a QuantStudio ${ }^{\text {TM }} 7$ Flex Real-Time PCR System with the primers specific for SARS-CoV-2 E gene ${ }^{43}$. For absolutely quantification of E gene RNA copies, a standard curve was generated using dilutions viral RNA of known copy number. E gene copies per ml of original virus supernatant were then calculated using this standard curve. 
bioRxiv preprint doi: https://doi.org/10.1101/2021.12.31.474653; this version posted January 3, 2022. The copyright holder for this preprint (which was not certified by peer review) is the author/funder, who has granted bioRxiv a license to display the preprint in perpetuity. It is made available under aCC-BY-NC 4.0 International license.

To measure the proportions of Omicron and Delta RNA from mixed samples a modified RTqPCR protocol was developed using probes specific for the $S$ gene of Omicron or Delta variants. Flanking primers that were used that were able to recognise a region of both Delta and Omicron (Forward: TGGACCTTGAAGGAAAACAGGG and Reverse: TGGTTCTAAAGCCGAAAAACCC). A pair of probes were then designed - a FAM probe specific to a 9 base pair insertion/deletion in the Omicron S gene at amino acid position 214 (TTATAGTGCGTGAGCCAGAAGA), and a VIC probe specific to the same region of the Delta $S$ gene sequence lacking this insertion/deletion (CTATTAATTTAGTGCGTGATCT). Reactions were run on AgPath RT-PCR (Life Technologies) kit on a QuantStudio $^{\text {TM }} 7$ Flex Real-Time PCR System and the relative amounts of FAM and VIC signal used to determine the proportion of Omicron and Delta in the original samples. Validation of the assay was carried out by extracting RNA from pure Omicron and Delta supernatants, normalising by $\mathrm{E}$ gene copies and mixing at different ratios before measuring using the assay.

\section{Acknowledgements and funding}

The authors would like to thank Dr Matthew Turnbull and Dr Suzannah Rihn of the MRCUniversity of Glasgow Centre for Virus Research (CVR) for sharing their Vero E6-ACE2-TMPRSS2 cells and Gavin Screaton, Wanwisa Dejnirattisai and Alison Cowper from Oxford University for sharing the Omicron isolate. For the other Delta and Omicron swab samples we thank Thushan de Silva at University of Sheffield, the ISARIC4C consortium and Paul Randell, Marcus Pond and colleagues at NWLP and PHE.

This work was supported by the G2P-UK National Virology Consortium funded by the MRC (MR/W005611/1). Additional funding to DB, NT and JN were funded by The Pirbright Institute's BBSRC institute strategic programme grant (BBS/E/I/00007038).

\section{References}

1 Viana, R. et al. Rapid epidemic expansion of the SARS-CoV-2 Omicron variant in southern Africa. medRxiv, 2021.2012.2019.21268028, doi:10.1101/2021.12.19.21268028 (2021).

$2 \quad$ UK Health Security Agency. Technical briefing 33. (UK HSA, 2021).

3 Cele, S. et al. SARS-CoV-2 Omicron has extensive but incomplete escape of Pfizer BNT162b2 elicited neutralization and requires ACE2 for infection. medRxiv, 2021.2012.2008.21267417, doi:10.1101/2021.12.08.21267417 (2021).

$4 \quad$ Newman, J. et al. Neutralising antibody activity against SARS-CoV-2 variants, including Omicron, in an elderly cohort vaccinated with BNT162b2. medRxiv, 2021.2012.2023.21268293, doi:10.1101/2021.12.23.21268293 (2021).

5 Cameroni, E. et al. Broadly neutralizing antibodies overcome SARS-CoV-2 Omicron antigenic shift. bioRxiv, 2021.2012.2012.472269, doi:10.1101/2021.12.12.472269 (2021).

6 Cao, Y. et al. Omicron escapes the majority of existing SARS-CoV-2 neutralizing antibodies. bioRxiv, 2021.2012.2007.470392, doi:10.1101/2021.12.07.470392 (2021).

7 Planas, D. et al. Considerable escape of SARS-CoV-2 variant Omicron to antibody neutralization. bioRxiv, 2021.2012.2014.472630, doi:10.1101/2021.12.14.472630 (2021). 
8

9

10

11

12

Liu, L. et al. Striking Antibody Evasion Manifested by the Omicron Variant of SARS-CoV-2. bioRxiv, 2021.2012.2014.472719, doi:10.1101/2021.12.14.472719 (2021).

Hoffmann, M. et al. The Omicron variant is highly resistant against antibody-mediated neutralization - implications for control of the COVID-19 pandemic. Cell, doi:https://doi.org/10.1016/j.cell.2021.12.032 (2021).

Pulliam, J. R. C. et al. Increased risk of SARS-CoV-2 reinfection associated with emergence of the Omicron variant in South Africa. medRxiv, 2021.2011.2011.21266068, doi:10.1101/2021.11.11.21266068 (2021).

Ferguson, N. et al. Report 49 - Growth, population distribution and immune escape of Omicron in England. (2021).

Rihn, S. J. et al. A plasmid DNA-launched SARS-CoV-2 reverse genetics system and coronavirus toolkit for COVID-19 research. PLoS Biol 19, e3001091, doi:10.1371/journal.pbio.3001091 (2021).

Peacock, T. P. et al. The furin cleavage site in the SARS-CoV-2 spike protein is required for transmission in ferrets. Nat Microbiol, doi:10.1038/s41564-021-00908-w (2021).

UK Health Security Agency. Technical briefing 29. (UK HSA, 2021).

Zhou, J. et al. Mutations that adapt SARS-CoV-2 to mustelid hosts do not increase fitness in the human airway. bioRxiv, 2021.2008.2020.456972, doi:10.1101/2021.08.20.456972 (2021).

Dinnon, K. H. et al. A mouse-adapted model of SARS-CoV-2 to test COVID-19 countermeasures. Nature 586, 560-566, doi:10.1038/s41586-020-2708-8 (2020). Muruato, A. et al. Mouse Adapted SARS-CoV-2 protects animals from lethal SARS-CoV challenge. bioRxiv, doi:10.1101/2021.05.03.442357 (2021).

Hale, V. L. et al. SARS-CoV-2 infection in free-ranging white-tailed deer. Nature, doi:10.1038/s41586-021-04353-x (2021).

Thakur, N. et al. SARS-CoV-2 variants of concern Alpha, Beta, Gamma and Delta have extended ACE2 receptor host-ranges. bioRxiv, 2021.2011.2023.469663, doi:10.1101/2021.11.23.469663 (2021).

Mlcochova, P. et al. SARS-CoV-2 B.1.617.2 Delta variant replication and immune evasion. Nature, doi:10.1038/s41586-021-03944-y (2021).

Saito, A. et al. Enhanced fusogenicity and pathogenicity of SARS-CoV-2 Delta P681R mutation. Nature, doi:10.1038/s41586-021-04266-9 (2021).

Peacock, T. P. et al. The SARS-CoV-2 variants associated with infections in India, B.1.617, show enhanced spike cleavage by furin. bioRxiv, 2021.2005.2028.446163, doi:10.1101/2021.05.28.446163 (2021).

Winstone, H. et al. The polybasic cleavage site in the SARS-CoV-2 spike modulates viral sensitivity to Type I interferon and IFITM2. J Virol, doi:10.1128/JVI.02422-20 (2021). Hoffmann, M., Kleine-Weber, H. \& Pöhlmann, S. A Multibasic Cleavage Site in the Spike Protein of SARS-CoV-2 Is Essential for Infection of Human Lung Cells. Mol Cell 78, 779784.e775, doi:10.1016/j.molcel.2020.04.022 (2020).

Puray-Chavez, M. et al. Systematic analysis of SARS-CoV-2 infection of an ACE2-negative human airway cell. Cell Reports 36, 109364, doi:https://doi.org/10.1016/i.celrep.2021.109364 (2021). Heurich, A. et al. TMPRSS2 and ADAM17 cleave ACE2 differentially and only proteolysis by TMPRSS2 augments entry driven by the severe acute respiratory syndrome coronavirus spike protein. J Virol 88, 1293-1307, doi:10.1128/jvi.02202-13 (2014).

Muus, C. et al. Single-cell meta-analysis of SARS-CoV-2 entry genes across tissues and demographics. Nature Medicine 27, 546-559, doi:10.1038/s41591-020-01227-z (2021). Meng, B. et al. SARS-CoV-2 Omicron spike mediated immune escape, infectivity and cell-cell fusion. bioRxiv, 2021.2012.2017.473248, doi:10.1101/2021.12.17.473248 (2021). 
29 The Genotype to Phenotype Japan (G2P-Japan) Consortium. Attenuated fusogenicity and pathogenicity of SARS-CoV-2 Omicron variant. (2021).

30 Braga, L. et al. Drugs that inhibit TMEM16 proteins block SARS-CoV-2 spike-induced syncytia. Nature 594, 88-93, doi:10.1038/s41586-021-03491-6 (2021).

31 Johnson, B. A. et al. Furin Cleavage Site Is Key to SARS-CoV-2 Pathogenesis. bioRxiv, 2020.2008.2026.268854, doi:10.1101/2020.08.26.268854 (2020).

32 Bentley, E. G. et al. SARS-CoV-2 Omicron-B.1.1.529 Variant leads to less severe disease than Pango $B$ and Delta variants strains in a mouse model of severe COVID-19. bioRxiv, 2021.2012.2026.474085, doi:10.1101/2021.12.26.474085 (2021).

33 Abdelnabi, R. et al. The omicron (B.1.1.529) SARS-CoV-2 variant of concern does not readily infect Syrian hamsters. bioRxiv, 2021.2012.2024.474086, doi:10.1101/2021.12.24.474086 (2021).

34 Yin, W. et al. Structures of the Omicron Spike trimer with ACE2 and an anti-Omicron antibody. bioRxiv, 2021.2012.2027.474273, doi:10.1101/2021.12.27.474273 (2021).

$35 \mathrm{Ni}$, D. et al. Structural analysis of the Spike of the Omicron SARS-COV-2 variant by Cryo-EM and implications for immune evasion. bioRxiv, 2021.2012.2027.474250, doi:10.1101/2021.12.27.474250 (2021).

36 Conceicao, C. et al. The SARS-CoV-2 Spike protein has a broad tropism for mammalian ACE2 proteins. PLoS Biol 18, e3001016, doi:10.1371/journal.pbio.3001016 (2020).

37 McKay, P. F. et al. Self-amplifying RNA SARS-CoV-2 lipid nanoparticle vaccine candidate induces high neutralizing antibody titers in mice. Nat Commun 11, 3523, doi:10.1038/s41467-020-17409-9 (2020).

38 David, A. et al. A common <em>TMPRSS2</em> variant protects against severe COVID-19. medRxiv, 2021.2003.2004.21252931, doi:10.1101/2021.03.04.21252931 (2021).

39 Edie, S. et al. Survey of human chromosome 21 gene expression effects on early development in Danio rerio. G3: Genes, Genomes, Genetics 8, 2215-2223 (2018).

40 Thakur, N. et al. Micro-fusion inhibition tests: quantifying antibody neutralization of virusmediated cell-cell fusion. Journal of General Virology 102, doi:https://doi.org/10.1099/igv.0.001506 (2021).

41 Ishikawa, H., Meng, F., Kondo, N., Iwamoto, A. \& Matsuda, Z. Generation of a dual-functional split-reporter protein for monitoring membrane fusion using self-associating split GFP. Protein Eng Des Sel 25, 813-820, doi:10.1093/protein/gzs051 (2012).

42 Zhou, J. et al. Investigating SARS-CoV-2 surface and air contamination in an acute healthcare setting during the peak of the COVID-19 pandemic in London. Clin Infect Dis, doi:10.1093/cid/ciaa905 (2020).

43 Corman, V. M. et al. Detection of 2019 novel coronavirus (2019-nCoV) by real-time RT-PCR. Eurosurveillance 25, 2000045, doi:doi:https://doi.org/10.2807/15607917.ES.2020.25.3.2000045 (2020). 


\section{Supplementary Figures}

A

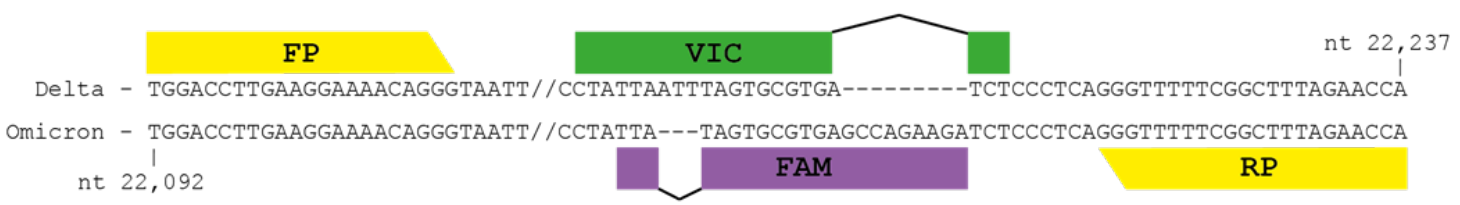

B

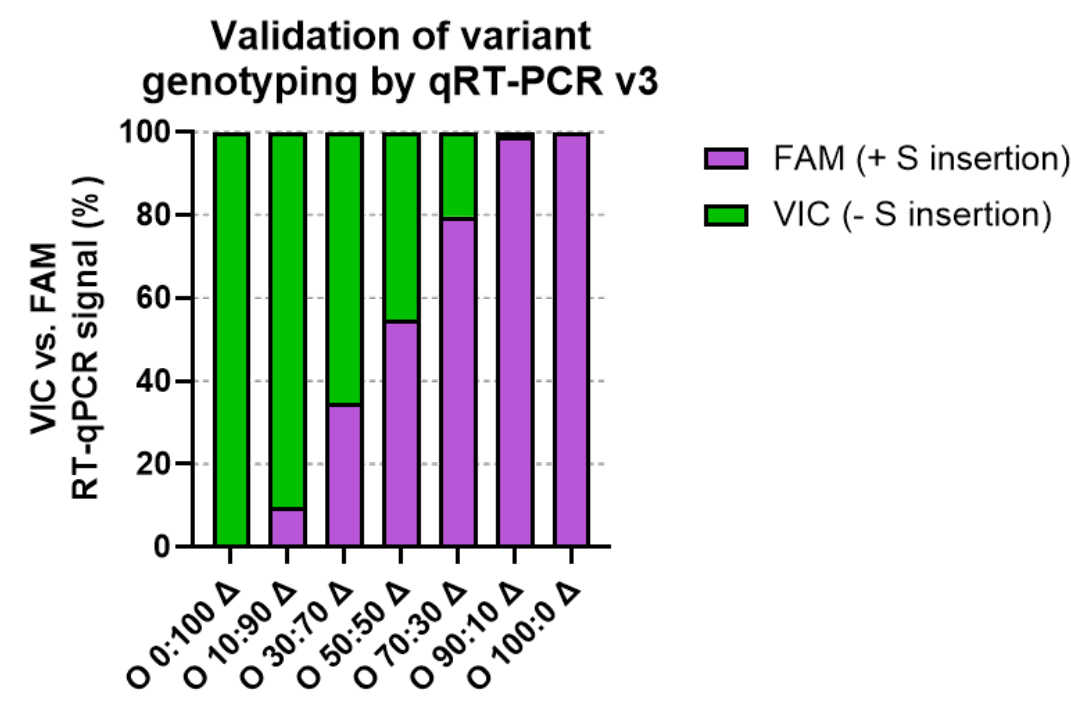

RNA mixture

Supplementary Figure S1 - Design and validation of variant specific multiplexed RT-qPCR and independent isolate repeats. (A) Design schematic of variant specific qPCR primer probe sets for investigating the ratio of Delta to Omicron in competition assays. (B) Different ratios of Delta and Omicron RNA were mixed and the variant specific RT-qPCR probes, which specifically recognise a region of Spike that includes a deletion and an insertion difference between Delta and Omicron were tested. 

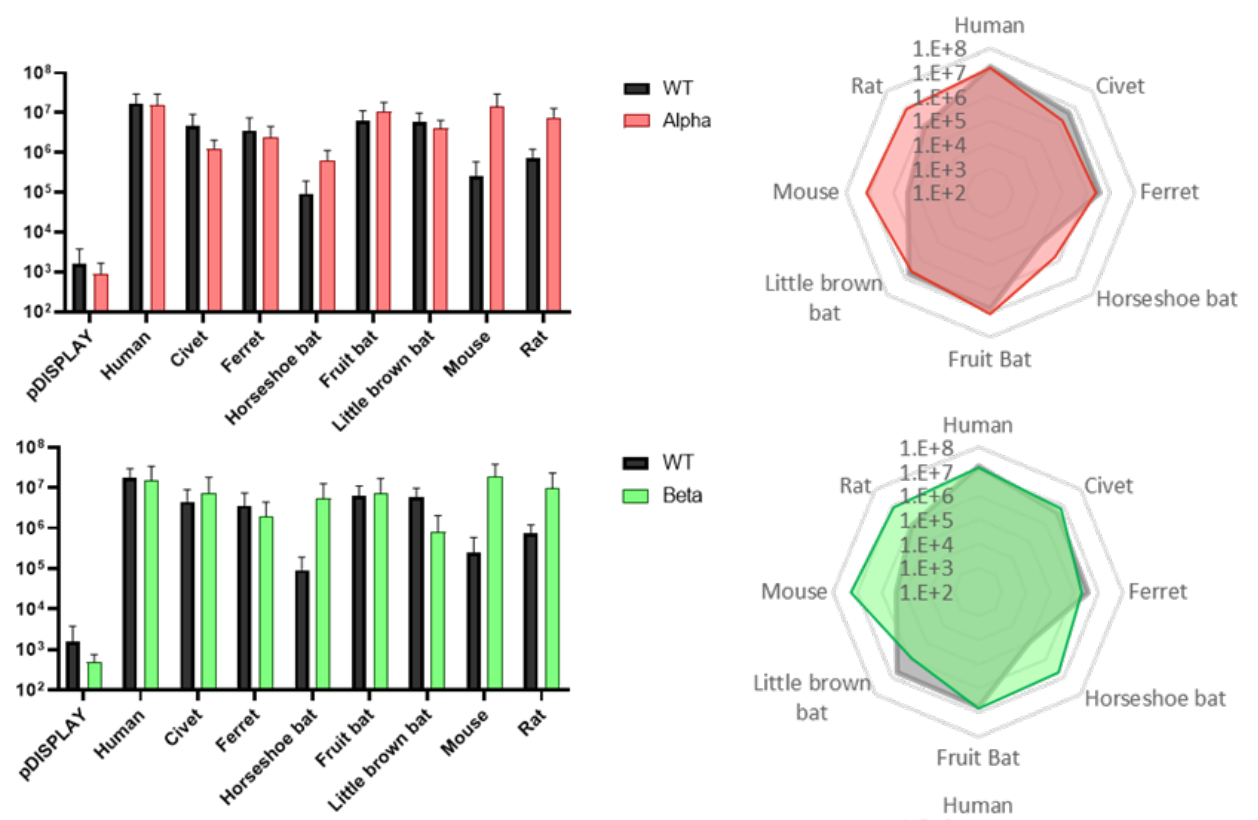

$\square$ WT
$\square$ Beta
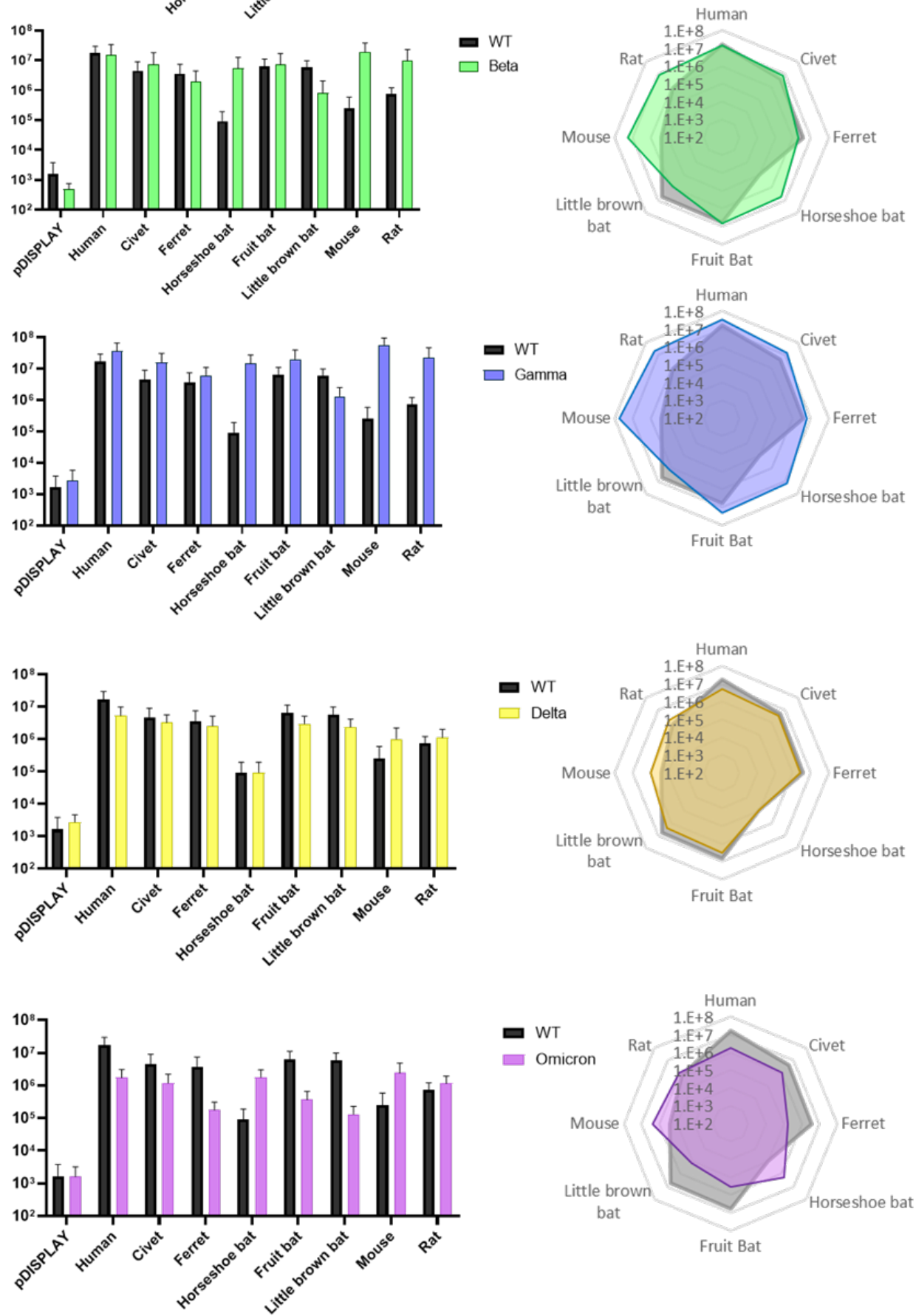

Supplementary Figure S2 - Different species ACE2 preference of different variants of concern. Receptor usage was screened using pseudoviruses expressing the indicated Spike proteins into 293Ts expressing the indicated ACE2 protein. Viral entry was measured by assaying luciferase activity (RLU) using the BrightGlo reagent (Promega). 